\title{
Atividade de Lipase em Quimo de Três Espécies Tropicais de Peixes Teleostei de Água Doce
}

\author{
José Teixeira Seixas Filho1 ${ }^{1,2}$, Maria Gorethi A. Oliveira ${ }^{3}$, Juarez Lopes Donzele ${ }^{4}$, Andréa T. M. \\ Gomide $^{5}$, Eliane Menin 6
}

\begin{abstract}
RESUMO - O objetivo do presente trabalho foi avaliar a atividade de lipase no quimo dos intestinos médio e posterior de três espécies de peixes Teleostei, com hábitos alimentares diferentes, piracanjuba (Brycon orbignyanus), piau (Leporinus friderici), onívoros, e surubim (Pseudoplatystoma curuscans), preferencialmente carnívoro, visando fornecer referência à nutrição para o ajuste de diferentes sistemas de alimentação artificial. Foram determinadas as atividades de lipase, utilizando-se kit BIOCLIN. Os resultados demonstraram atividade específica média de lipase de 99,86\%, sendo menor para a piracanjuba (1,89 UI/mg) em relação ao piau (1347,82 UI/mg), ambos de hábito alimentar onívoro. A diferença de atividade específica de lipase entre piracanjuba e surubim (793,76 UI/mg) também apresentou média de $99,76 \%$. O surubim apresentou atividade específica de lipase $41,11 \%$ menor que o piau. Estes dados sugerem que o piau apresenta atividade específica de lipase mais próxima à de um peixe carnívoro que onívoro. Estudos adicionais com testes de alimentação são necessários para a avaliação do comportamento destas espécies a novos sistemas de alimentação.
\end{abstract}

Palavras-chave: atividade de lipase, enzima, nutrição animal, piracanjuba, piau, surubim

\section{Lipase Activity in the Chime of Three Teleostei Freshwater Fish}

\begin{abstract}
The objective of this work was to evaluate the lipase activity in the chime present in the intestines or in the rectum of three tropical freshwater Teleostei species with different feeding habits: piracanjuba, Brycon orbignyanus (Valenciennes, 1849), piau, Leporinus friderici, omnivorous, and surubim (Pseudoplatystoma coruscans), preferably carnivorous, aiming to provide nutritional data for the adjustment of different artificial feeding systems. The lipase activity was thus determined by the use of BIOCLIN kit. The results showed average specific lipase activity of $99.86 \%$, being lower for piracanjuba ( $1.89 \mathrm{UI} / \mathrm{mg}$ ) as compared to piau (1347.82 UI/mg), both omnivorous, while the activity of the same enzyme for piracanjuba was $99.76 \%$ lower when compared to surubim (793.76 UI/mg). Surubim showed specific activity of $41.11 \%$ lower than of piau. This data suggests that piau presented specific lipase activity closely to carnivorous fish than the omnivorous one. Additional studies with feeding test are necessary to evaluate the behavior of these species under a new feeding systems.
\end{abstract}

Key Words: lipase activity, enzyme, animal nutritional, piracanjuba, piau, surubim

\section{Introdução}

Várias espécies tropicais de peixes Teleostei de água doce são criadas em cativeiro, tanto para manutenção da biodiversidade, por meio de programas de repovoamento, quanto para criação comercial. Contudo, essas espécies ainda recebem arraçoamento de maneira imprópria, por falta de maior entendimento sobre suas necessidades nutricionais.

Nesse sentido, estudos têm sido realizados para se conhecerem as necessidades nutricionais das espécies nativas com potencial zootécnico, com intuito de proporcionar às piscigranjas soluções ao menor ganho de peso, sinais de deficiência alimentar e variação na taxa de ingestão de alimentos, problemas que resultam em baixa produtividade e mortalidade elevada. Vários autores têm relatado sobre o hábito alimentar de peixes tropicais (CYRINO, 1984; CASTAGNOLLI, 1992, 1997; e PEZZATO, 1997) e o relacionamento do arranjo do intestino com hábitos alimentares (ALHUSSAINI, 1947, 1949; NIKOLSKY, 1963; PREJS,

\footnotetext{
1 Parte da Tese de Doutorado do primeiro autor, financiada pelo CNPq e pela FAPEMIG.

2 Pesquisador da Fund. Inst. de Pesca do Estado do RJ - FIPERJ - Alameda São Boaventura, 770, Fonseca, Niterói, RJ 24120 -191.

3 Profa do Dpto de Bioq. e Biol. Molecular e BIOAGRO/UFV, Viçosa - 36.571-000, MG.

4 Professor do Dpto de Zootecnia/UFV, Viçosa - 36.571.000, MG.

5 Zootecnista, Bolsista de DTI do DBA/UFV, Viçosa - 36571-000, MG.

6 Professor do Dpto. de Biologia Animal/ UFV, Viçosa - 36.571.000, MG.
} 
1981; MENIN, 1988; MENIN e MIMURA, 1992; e SEIXAS FILHO, 1998). A habilidade de um organismo para digerir partículas de alimento depende da presença e da quantidade apropriada de enzimas digestivas (SMITH, 1980). A atividade enzimática de lipase (EC 3.1.1.3) para os peixes de clima temperado durante as diferentes fases do ciclo de vida tem sido pesquisada (ANDREWS, 1954; KITAMIKADO e TACHINO, 1961; KAWAI e IKEDA, 1972; CHESLEY, 1934; ABI-AYAD e KESTEMONT, 1994; INFANTE e CAHU, 1994; SASTRY, 1974 a, b; NAKAGAWA et al., 1995; e SABAPATHY e TEO, 1993).

CHESLEY (1934) relatou que não houve correlação entre o conteúdo de gordura da alimentação fornecida ao peixe e a atividade de lipase. NAGASE(1964), estudando a tilápia mossambica (Sarotherodon mossambicus), observou que não houve aumento na atividade de lipase, quando este recebeu dieta rica em gordura, concordando com AGRAWAL et al. (1975), que mencionaram não haver correlação da atividade de lipase com a gordura da dieta. Por outro lado, REIMER (1982), estudando o matrinchã (Brycon cf. melanopterus), concluiu que o padrão enzimático de lipase é ajustado à quantidade de gordura presente no conteúdo intestinal.

BORLONGAN (1990) relatou que descobertas histoquímicas recentes mostraram que a mucosa intestinal de diversos peixes Teleostei é capaz de secretar lipase, além do pâncreas difuso, compensando a falta de um pâncreas compacto e bem desenvolvido. $\mathrm{O}$ mesmo autor observou em "milkfish" (Chanos chanos) atividade de lipase no esôfago, indicando um papel mais ativo desse órgão no processo digestório.

KUZ'MINA (1996) estudou a influência da idade na atividade enzimática em peixes de água doce, predadores facultativos bentofágicos adultos, como a perca, assim como nos bentofágicos, Bream e Roach. Esse autor mencionou que a atividade enzimática total relativa ( $\mathrm{g}^{-1}$ peso do corpo) é, para o nutricionista, índice informativo do suprimento de enzimas digestivas em peixes mais importante que a atividade enzimática padrão normalmente relatada.

O objetivo do presente trabalho foi avaliar a atividade enzimática da lipase no quimo presente nos intestinos médio e posterior de peixes Teleostei, com hábitos alimentares diferentes, piracanjuba (Brycon orbignyanus), piau (Leporinus friderici), onívoros e surubim (Pseudoplatystoma coruscans), carnívoro, visando fornecer referência à nutrição para o ajuste de diferentes sistemas de alimentação artificial.

\section{Material e Métodos}

Os exemplares, de piracanjuba, piau e surubim, utilizados nos estudos de atividade enzimática, foram coletados dos viveiros da Estação de Pesquisas e Desenvolvimento Ambiental de Volta Grande CEMIG, Conceição das Alagoas, MG, com auxílio de redes de arrasto. A água dos viveiros apresentou temperatura de $24,5^{\circ} \mathrm{C}$, pH de 6,52 , oxigênio dissolvido de $8,67 \mathrm{ppm}$ e condutividade de $0,032 \mathrm{~ms} / \mathrm{cm}$.

\section{Coleta das amostras}

Os animais foram submetidos à intensa alimentação 24 horas antes da captura, sendo que os peixes onívoros receberam ração comercial (Tabela 1) e os carnívoros, pedaços de peixe e juvenis de tilápia vivos.

Dez exemplares de cada espécie foram imediatamente mortos após a captura por meio de secção transversal da medula espinhal, imediatamente após a região occipital, sendo imersos em água a $4{ }^{\circ} \mathrm{C}$. Os animais foram submetidos à essa temperatura durante todo o período em que foram manuseados. Em seguida, os exemplares foram transferidos para o laboratório da Estação, identificados e submetidos às medidas de peso e comprimento-padrão, que consistiu na medida da ponta do focinho até a inserção dos raios da nadadeira caudal, conforme RICKER (1968).

Isolamento do tubo digestivo e dos segmentos a serem analisados

Os animais sofreram incisão longitudinal ventral, procedendo-se à retirada da gordura que envolvia o tubo digestivo, cujo isolamento foi feito após ligaduras duplas na porção cranial do esôfago e na porção caudal do reto, anteriormente ao ânus. Além dessas ligaduras, outras foram realizadas antes dos intestinos médio e posterior, para evitar o extravasamento do quimo das regiões a serem estudadas.

Os segmentos trabalhados, padronizados por medida a partir do esfíncter pilórico, foram o cranial, mediano e caudal do intestino médio; cranial e caudal do intestino posterior. As amostras do quimo dos segmentos compuseram um "pool", sendo retiradas, pesadas, acondicionadas em embalagem plástica e armazenadas $\mathrm{a}-4^{\circ} \mathrm{C}$.

Preparação das amostras para as análises de atividade enzimática

O quimo dos intestinos médio e posterior de cada exemplar do piau, obtido em pequena quantidade, foi coletado e congelado em frascos. Em seguida, o 
Tabela 1 - Ração comercial utilizada na alimentação dos exemplares de Brycon orbignyanus e Leporinus friderici

Table 1 - Commercial diet used to Brycon orbignyanus and Leporinus friderici species feeding

\begin{tabular}{lc}
\hline $\begin{array}{l}\text { Nível de garantia (\%) } \\
\text { Guarantee level }\end{array}$ & $\begin{array}{c}\text { Ração comercial } \\
\text { Commercial diet }\end{array}$ \\
\hline $\begin{array}{l}\text { Umidade (máxima) } \\
\text { Humidity (maximum) }\end{array}$ & 13,00 \\
$\begin{array}{l}\text { Proteína bruta (PB) (mínimo) } \\
\text { Crude protein (CP) (minimum) }\end{array}$ & 28,00 \\
$\begin{array}{l}\text { Estrato etéreo (mínimo) } \\
\text { Ether extract (minimum) } \\
\text { Matéria fibrosa (máximo) }\end{array}$ & 3,00 \\
$\begin{array}{l}\text { Fibrous matter (maximum) } \\
\text { Material mineral (máximo) } \\
\text { Mineral material (maximum) }\end{array}$ & 9,00 \\
$\begin{array}{l}\text { Cálcio(máximo) } \\
\text { Calcium (maximum) }\end{array}$ & 10,00 \\
$\begin{array}{l}\text { Fósforo (P) } \\
\text { Phosphorus }\end{array}$ & 1,60 \\
Comporato & 0,60
\end{tabular}

Composição básica $\quad$ Eventuais substitutos

Basic composition Substitute eventuals

\begin{tabular}{lc}
\hline Milho & Sorgo \\
Corn & Sorghum \\
Farelo de soja & Farinha de sangue
\end{tabular}

Soybean meal

Farelo de glúten de milho $21 \%$

Corn gluten meal $21 \%$

Farelo de glúten de milho $60 \%$

Corn gluten meal $60 \%$

Farinha de peixe

Fish meal

Farinha de carne e ossos

Meat and bone meal

Levedura de álcool de cana

Sugarcane alcohol yeast

Óleo de soja

Soybean oil

Sal

Salt

Carbonato de cálcio

Calcium carbonate

Sal Suplemento Vit. ${ }^{1}$ e mineral ${ }^{2}$

Salt supplement with vit. ${ }^{1}$ and minerals ${ }^{2}$

Antioxidante $^{3}$

Antioxidant ${ }^{3}$

${ }^{1}$ Composição por kg da dieta (Composition per kg of diet): Vit. A, $12.000 \mathrm{UI}$; Vit $\mathrm{D}_{3}, 2000 \mathrm{UI}$; Vit. E, $15 \mathrm{Ul}$; Vit. K, $2 \mathrm{mg}$; Tiamina (Tiamin), $1,5 \mathrm{mg}$; Riboflavina (Riboflavin), $6 \mathrm{mg}$; Niacina (Niacin), $35 \mathrm{mg}$; Ác. pantotênico (Panthothenic acid), $11 \mathrm{mg}$; Piridoxina ( $\left.\mathrm{B}_{6}\right)$ (Pyridoxine), 2,5 mg; Colina (Choline), $350 \mathrm{mg}$; Ác. fólico (Folic acid), 0,6 mg; Biotina (Biotin), $100 \mathrm{mg}$; e Vit. B $12,15 \mu \mathrm{g}$.

2 Composição por kg da dieta (Composition perkg of diet): Zn, $60 \mathrm{mg}$; Mn, 70 mg; Cu, 8 mg; Fe, 30 mg; I, 1 mg; Co, 0,1 mg; e Se, 0,2 mg.

3 Etoxiquina (Ethoxiquin) - $250 \mathrm{mg}$. material foi colocado em "ependorff", sendo congelado e armazenado em freezer. Para as análises, o material foi descongelado em banho de gelo, centrifugado (35.000g, $4^{\circ} \mathrm{C}, 20$ minutos) e o sobrenadante utilizado para determinação da atividade de lipase.

Nos exemplares de piracanjuba e surubim, encontrou-se maior quantidade de quimo. Procedeu-se, então, ao descongelamento das amostras em banho de gelo e à pesagem de $0,125 \mathrm{mg}$ de quimo, diluído em $2,5 \mathrm{~mL}$ de água destilada congelada, triturado e homogeneizado com auxílio de bastão de vidro. Após esse processo, foi centrifugado como descrito anteriormente.

Atividade da lipase

As atividades foram determinadas por intermédio de um kit BIOCLIN, com metodologia modificada de CHERRY (1932), que consiste na verificação da atuação da lipase do quimo sobre um éster de glicerol, liberando um cromogênio, que é quantitativamente determinado em $410 \mathrm{~nm}$. A intensidade de cor formada é proporcional à atividade de lipase e os valores são expressos em Unidades Internacionais (UI).

Determinação da concentração de proteína do quimo

A determinação da concentração de proteína foi realizada por meio da leitura de absorção a $260 \mathrm{e}$ $280 \mathrm{~nm}$, segundo método descrito por WARBURG e CHRISTIAN (1941).

Determinação da atividade específica da lipase

Os valores da atividade de lipase foram divididos pela proteína total corrigida, a qual foi obtida após a correção da presença de resíduos de tirosina, triptofano e ácidos nucléicos, quando da aplicação do Método de WARBURG e CHRISTIAN (1941), resultando na atividade específica da enzima.

\section{Análise estatística}

As médias foram comparadas pelo teste " $\mathrm{t}$ ", em nível de 5\% de probabilidade.

\section{Resultados e Discussão}

As médias dos pesos e comprimentos dos exemplares de piracanjuba, piau e surubim encontram-se na Tabela 2. Constatou-se que os exemplares de piau apresentaram menor $(\mathrm{P}<0,05)$ peso corporal em rela- 
ção aos exemplares de piracanjuba e surubim, cujos pesos não variaram $(\mathrm{P}>0,05)$ entre si, e menor $(\mathrm{P}<0,05)$ valor de comprimento-padrão em relação ao surubim. O comprimento-padrão apresentado pelos exemplares de piracanjuba foi menor $(\mathrm{P}<0,05)$ que o do surubim e não variou $(\mathrm{P}>0,05)$ em relação ao apresentado pelo piau, embora tenha sido observada diferença de $49,8 \%$ entre esses valores.

Observou-se variação na atividade específica de lipase no quimo dos exemplares de piracanjuba, sendo a menor de $0,694 \mathrm{UI} / \mathrm{mg}$ no exemplar que apresentou peso e comprimento de $381,21 \mathrm{~g}$ e $23,50 \mathrm{~cm}$, respectivamente (Tabela 3). A maior atividade foi de $2,609 \mathrm{UI} / \mathrm{mg}$ para o exemplar que apresentou peso de $601,54 \mathrm{~g}$ e $29,50 \mathrm{~cm}$ de comprimento.

Foi verificada a atividade por peso e comprimento do animal, com variação de $0,1301 \times 10^{-4} \mathrm{UI} / \mathrm{g}$ a 8,980 x $10^{-4} \mathrm{UI} / \mathrm{g}$ e $1,005 \times 10^{-2} \mathrm{UI} / \mathrm{cm}$ a 92,629 x $10^{-2} \mathrm{UI} / \mathrm{cm}$, respectivamente (Tabela 3 ).

Para os exemplares de piau, a atividade específica de lipase variou de 333,00 UI/mg, para o exemplar com peso e comprimento de $111,54 \mathrm{~g}$ e $17,00 \mathrm{~cm}$, respectivamente, a 1.700,00 UI/mg, para o com peso de $151,07 \mathrm{~g}$ e comprimento de $19,00 \mathrm{~cm}$. A menor atividade por peso e comprimento foi de $0,500 \mathrm{UI} / \mathrm{g} \mathrm{e}$ $3,346 \mathrm{UI} / \mathrm{cm}$, respectivamente, no exemplar com $113,71 \mathrm{~g}$ e $17,00 \mathrm{~cm}$. A maior atividade por peso e comprimento foi de 2,103 UI/g no exemplar com $96,06 \mathrm{~g}$ e $16,50 \mathrm{~cm}$ e $13,839 \mathrm{UI} / \mathrm{cm}$ no peixe com $161,70 \mathrm{~g}$ e 19,00 cm, respectivamente (Tabela 4).

Nos exemplares de surubim (Tabela 5), a atividade específica variou de $195,996(226,83 \mathrm{~g} \mathrm{e} 30,00 \mathrm{~cm})$ a $1957,689 \mathrm{UI} / \mathrm{mg}(630,33 \mathrm{~g} \mathrm{e} 42,00 \mathrm{~cm})$ e a atividade por peso e comprimento, de $0,072 \mathrm{UI} / \mathrm{g}$ e $0,830 \mathrm{UI} / \mathrm{cm}$ $(440,75 \mathrm{~g}$ e $38,00 \mathrm{~cm})$ a $0,926 \mathrm{UI} / \mathrm{g}$ e $9,428 \mathrm{UI} / \mathrm{cm}$ $(336,10 \mathrm{~g}$ e $33,00 \mathrm{~cm})$, respectivamente.

Pelas médias da atividade específica de lipase das três espécies (Tabela 6), pode-se observar maiores atividades para o piau $(1347,824 \mathrm{UI} / \mathrm{mg})$, seguida pela do surubim $(793,765 \mathrm{UI} / \mathrm{mg})$, que apresentaram diferenças significativas entre si $(\mathrm{P}<0,05)$. Por outro lado, o piracanjuba apresentou menor média de atividade específica, em relação às outras espécies, ou seja, 1,886 UI/mg, o que diferiu $(\mathrm{P}<0,05)$ do piau e surubim. Para a relação da atividade específica com o peso e o comprimento, todas apresentaram diferença significativa $(\mathrm{P}<0,05)$ entre si.

O perfil de atividade enzimática, concentração de proteína e atividades específicas de lipase no quimo de piracanjuba, piau e surubim encontra-se representado nas Figuras 1, 2 e 3, respectivamente. As
Tabela 2 - Média ( \pm erro-padrão da média) do peso e comprimento dos exemplares de piracanjuba, Brycon orbignyanus, piau, Leporinus friderici, e surubim, Pseudoplatystoma coruscans

Table 2 - Average ( \pm standard error) of weight and length of piracanjuba, Brycon orbignyanus, piau, Leporinus friderici, and surubim, Pseudoplatystoma coruscans species

\begin{tabular}{lcc}
\hline $\begin{array}{l}\text { Espécie } \\
\text { Specie }\end{array}$ & $\begin{array}{c}\text { Peso }(\mathrm{g}) \\
\text { Weight }\end{array}$ & $\begin{array}{c}\text { Comprimento-padrão }(\mathrm{cm}) \\
\text { Standard length }\end{array}$ \\
\hline Piracanjuba & $457,46( \pm 26,41) \mathrm{a}$ & $26,60( \pm 0,57) \mathrm{b}$ \\
Piau & $127,84( \pm 7,19) \mathrm{b}$ & $17,75( \pm 0,33) \mathrm{bc}$ \\
Surubim & $442,45( \pm 46,36) \mathrm{a}$ & $36,55( \pm 1,49) \mathrm{a}$ \\
CV $(\%)$ & 52,94 & 30,85 \\
\hline
\end{tabular}

Em cada coluna, médias seguidas pelas mesmas letras não diferem pelo teste $t$, a $5 \%$ de probabilidade.

$\mathrm{n}=10$.

In each column, means followed by the same letters do not differ by $t$ test at $5 \%$ of probability.
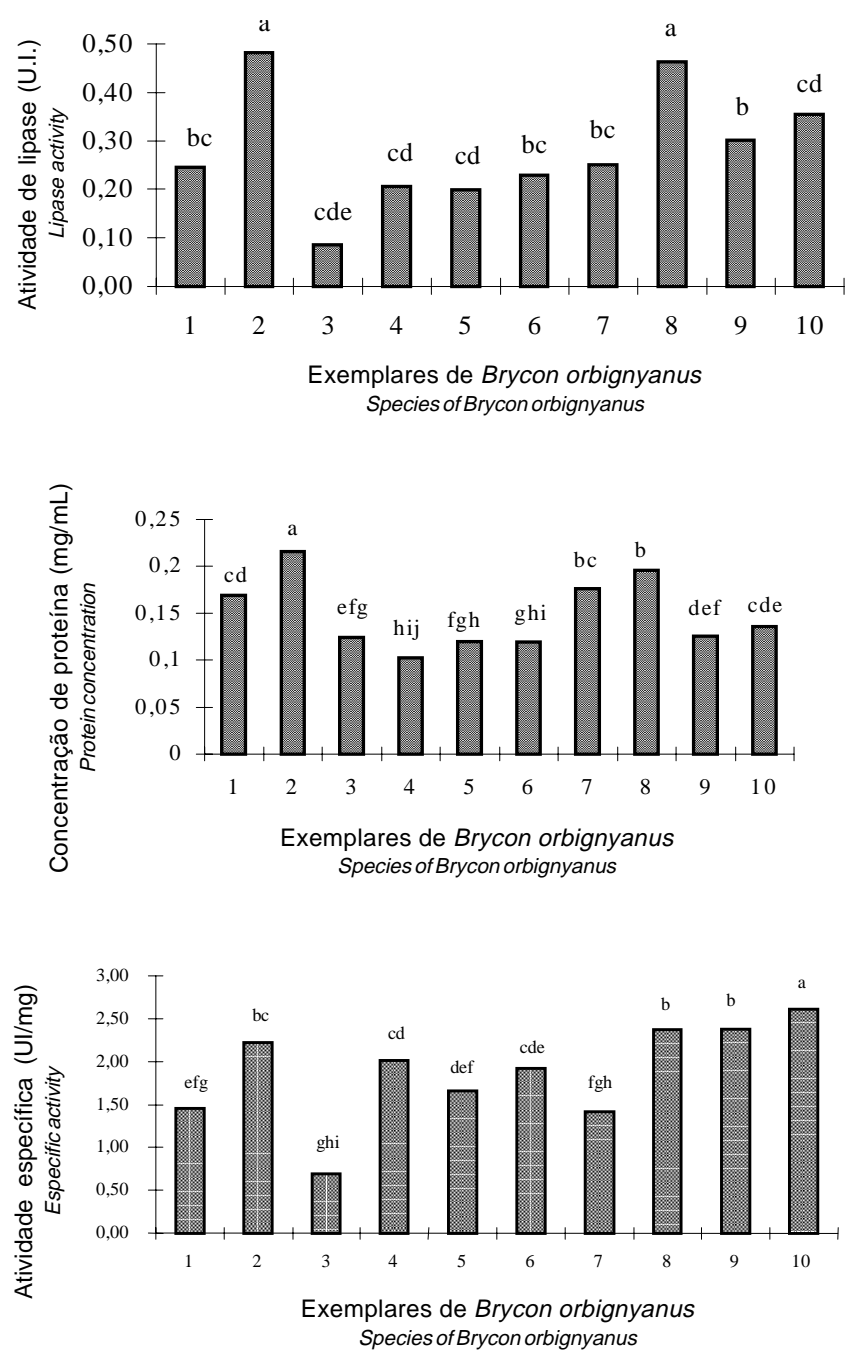

Figura 1 - Perfil das atividades, concentração de proteína e atividades específicas de lipase no quimo dos exemplares de piracanjuba, Brycon orbignyanus. Médias seguidas pelas mesmas letras não diferem pelo teste $t$, a $5 \%$ de probabilidade.

Figure 1 - Activity profile, protein concentration and lipase specific activity in the chyme of piracanjuba, Brycon orbignyanus species.

Means followed by the same letter do not differ by $t$ test, at $5 \%$ probability. 
10 Rev. bras. zootec.

Tabela 3 - Variação da atividade específica de lipase no quimo e sua relação com o peso e o comprimento dos exemplares de piracanjuba, Brycon orbignyanus

Table 3 - Lipase specific activity variation in the chyme and its relation to weigth and length of piracanjuba, Brycon orbignyanus species

\begin{tabular}{|c|c|c|c|c|c|}
\hline $\begin{array}{l}\text { Piracanjuba } \\
\text { (exemplar) } \\
\text { (species) }\end{array}$ & $\begin{array}{l}\text { Peso } \\
(\mathrm{g}) \\
\text { Weight }\end{array}$ & $\begin{array}{c}\text { Comprimento } \\
(\mathrm{cm}) \\
\text { Length }\end{array}$ & $\begin{array}{c}\text { Atividade } \\
\text { específica } \\
\text { (U.I./mg) } \\
\text { Specific activity }\end{array}$ & $\begin{array}{l}\text { Atividade/ } \\
\text { peso vivo (g) } \\
\text { (U.I./g) } \\
\text { Activity/ } \\
\text { live weight }\end{array}$ & $\begin{array}{c}\text { Atividade/ } \\
\text { comprimento }(\mathrm{cm}) \\
\text { (U.I./ } \mathrm{cm}) \\
\text { Activity/ } \\
\text { live length }\end{array}$ \\
\hline 01 & 351,99hij & $24,50 \mathrm{~cd}$ & $1,453 \mathrm{efg}$ & $6,997 \times 10^{-4} \mathrm{~b}$ & $1,005 \times 10^{-2} \mathrm{hij}$ \\
\hline 02 & $369,83 \mathrm{ghi}$ & $25,50 \mathrm{~cd}$ & $2,219 b c$ & $0,1301 \times 10^{-4} \mathrm{fgh}$ & $1,887 \times 10^{-2} \mathrm{def}$ \\
\hline 03 & $381,21 \mathrm{fgh}$ & $23,50 \mathrm{~cd}$ & 0,694ghi & $2,249 \times 10^{-4} \mathrm{efg}$ & $36,489 \times 10^{-2} \mathrm{cde}$ \\
\hline 04 & 405,60 efg & $27,50 \mathrm{bc}$ & $2,009 \mathrm{~cd}$ & $5,069 \times 10^{-4} \mathrm{cde}$ & $74,764 \times 10^{-2} \mathrm{bcd}$ \\
\hline 05 & $442,77 \mathrm{def}$ & $26,50 \mathrm{bc}$ & $1,658 \mathrm{def}$ & $4,495 \times 10^{-4} \mathrm{def}$ & $75,113 \times 10^{-2} \mathrm{bc}$ \\
\hline 06 & $457,58 \mathrm{cde}$ & $27,50 \mathrm{bc}$ & $1,921 \mathrm{cde}$ & $4,990 \times 10^{-4} \mathrm{cde}$ & $83,036 \times 10^{-2} \mathrm{~b}$ \\
\hline 07 & $491,00 \mathrm{~cd}$ & $27,00 \mathrm{bc}$ & $1,417 \mathrm{efg}$ & $5,093 \times 10^{-4} \mathrm{cde}$ & $92,629 \times 10^{-2} \mathrm{a}$ \\
\hline 08 & $515,72 b c$ & $26,00 \mathrm{bc}$ & $2,367 b$ & $8,980 \times 10^{-4} \mathrm{a}$ & $1,781 \times 10^{-2} \mathrm{efg}$ \\
\hline 09 & $557,32 \mathrm{~b}$ & $28,50 \mathrm{~b}$ & $2,379 b$ & $5,402 \times 10^{-4} \mathrm{~cd}$ & $1,056 \times 10^{-2} \mathrm{ghi}$ \\
\hline 10 & $601,54 a$ & $29,50 \mathrm{a}$ & $2,609 a$ & $5,896 \times 10^{-4} \mathrm{bc}$ & $1,202 \times 10^{-2} \mathrm{fgh}$ \\
\hline $\mathrm{CV}(\%)$ & 17,75 & 6,76 & 30,42 & 41,91 & 76,91 \\
\hline
\end{tabular}

Em cada coluna, médias seguidas pelas mesmas letras não diferem pelo teste t, a $5 \%$ de probabilidade. In each column, means followed by the same letters do not differ by t test at $5 \%$ probability.

Tabela 4 - Variação da atividade específica de lipase no quimo e sua relação com o peso e o comprimento dos exemplares de piau, Leporinus friderici

Table 4 - Lipase specific activity variation in the chyme and its relation to weigth and length of piau, Leporinus friderici species

\begin{tabular}{|c|c|c|c|c|c|}
\hline $\begin{array}{l}\text { Piau } \\
\text { (exemplar) } \\
\text { (species) }\end{array}$ & $\begin{array}{c}\text { Peso } \\
(\mathrm{g}) \\
\text { Weight }\end{array}$ & $\begin{array}{c}\text { Comprimento } \\
\text { (cm) } \\
\text { Length }\end{array}$ & $\begin{array}{c}\text { Atividade } \\
\text { específica } \\
\text { (U.I./mg) } \\
\text { Specific activity }\end{array}$ & $\begin{array}{c}\text { Atividade/ } \\
\text { peso vivo (g) } \\
\text { (U.I./g) } \\
\text { Activity/ } \\
\text { live weight } \\
\end{array}$ & $\begin{array}{c}\text { Atividade/ } \\
\text { comprimento }(\mathrm{cm}) \\
\text { (U.I./ } \mathrm{cm}) \\
\text { Activity/ } \\
\text { live length } \\
\end{array}$ \\
\hline 01 & 96,06ghi & $16,50 \mathrm{~b}$ & $1615,00 \mathrm{~b}$ & $2,103 a$ & $12,244 b$ \\
\hline 02 & $106,99 \mathrm{fgh}$ & $16,50 \mathrm{~b}$ & $529,00 f g h$ & $0,556 \mathrm{fgh}$ & 3,606fgh \\
\hline 03 & $109,52 \mathrm{efg}$ & $17,00 \mathrm{~b}$ & 660,00 efg & $1,883 b$ & $12,130 \mathrm{~b}$ \\
\hline 04 & $111,54 \mathrm{def}$ & $17,00 \mathrm{~b}$ & $333,00 \mathrm{hij}$ & $0,564 \mathrm{efg}$ & 3,703efg \\
\hline 05 & $113,71 \mathrm{cde}$ & $17,00 \mathrm{~b}$ & $375,00 \mathrm{ghi}$ & $0,500 \mathrm{hij}$ & 3,346ghi \\
\hline 06 & $138,78 \mathrm{~cd}$ & $18,50 \mathrm{a}$ & $1079,00 \mathrm{bcd}$ & $1,455 \mathrm{bc}$ & $10,92 b c$ \\
\hline 07 & $139,67 \mathrm{~cd}$ & $18,50 \mathrm{a}$ & $871,00 \mathrm{cde}$ & 0,990 cde & $7,475 \mathrm{def}$ \\
\hline 08 & $149,31 b c$ & $18,50 \mathrm{a}$ & $1160,00 \mathrm{bc}$ & $0,963 \mathrm{def}$ & $7,776 \mathrm{cde}$ \\
\hline 09 & $151,07 \mathrm{~b}$ & $19,00 \mathrm{a}$ & $1700,00 \mathrm{a}$ & $1,209 \mathrm{~cd}$ & $9,620 \mathrm{bcd}$ \\
\hline 10 & $161,70 \mathrm{a}$ & $19,00 \mathrm{a}$ & 833,97def & 0,530ghi & 13,839a \\
\hline $\mathrm{CV}(\%)$ & 16,97 & 84,78 & 30,42 & 41,991 & 76,91 \\
\hline
\end{tabular}

Em cada coluna, médias seguidas pelas mesmas letras não diferem pelo teste $t$, a $5 \%$ de probabilidade.

In each column, means followed by the same letters do not differ by t test at $5 \%$ probability.

diferenças significativas observadas $(\mathrm{P}<0,05)$ da atividade enzimática no quimo entre os exemplares das espécies em estudo refletiram os diferentes momentos de digestão em que estes se encontravam. Observou-se, após a correção com a concentração de proteína, atividade específica média de $99,86 \%$ menor $(1,886 \mathrm{UI} / \mathrm{mg})$ para lipase do piracanjuba em relação à do piau (1347,824 UI/mg), ambos de hábito alimentar onívoro, enquanto a atividade para o piracanjuba foi
99,76\% menor em relação ao surubim (793,765 UI/mg), carnívoro piscífago, que apresentou atividade específica de $41,11 \%$ menor em relação à do piau.

A variação da atividade específica de lipase nos exemplares da mesma espécie, provavelmente, esteve relacionada à quantidade de substrato ingerido (alimentação) e ao intervalo de tempo de administração de cada um, uma vez que foi coletado um "pool" de quimo dos intestinos médio e posterior (piracanjuba 
e piau) e intestino médio e reto de surubim, em razão da pouca quantidade de material, de acordo com as características morfométricas intestinais de cada espécie, principalmente o piau, que apresentou o menor calibre dos intestinos.

A observação de maiores valores para a média da atividade específica de lipase do piau em relação à do surubim, carnívoro piscífago, e a grande diferença entre esta espécie e piracanjuba, ambas onívoras, podem ser atribuídas às condições de criação em que se encontrava o animal, sugerindo plasticidade do complexo enzimático dessa espécie.

Em relação aos peixes de hábito alimentar onívoro, observou-se que o fato de a média da atividade específica de lipase do piau ter se apresentado maior que a encontrada para a outra espécie onívora em questão, o piracanjuba, nas condições em que foi conduzido este trabalho, pode ter sido influenciado pelo nível de lipídeos da dieta, conforme observado por REIMER (1982), que, trabalhando com Brycon cf melanopteru, encontrou correlação da quantidade de gordura da ração com a atividade de lipase, se não fosse o fato de ter sido administrada a mesma ração para as duas espécies em questão. Por outro lado, esses resultados também não se assemelham aos de CHESLEY (1934), NAGASE (1964) e AGRAWALL (1975), uma vez que houve resposta metabólica diferenciada entre as espécies onívoras em estudo.

Portanto, os dados obtidos permitem que se façam algumas inferências sobre a fisiologia digestiva do piau, ou seja, esta espécie possui maior potencial para
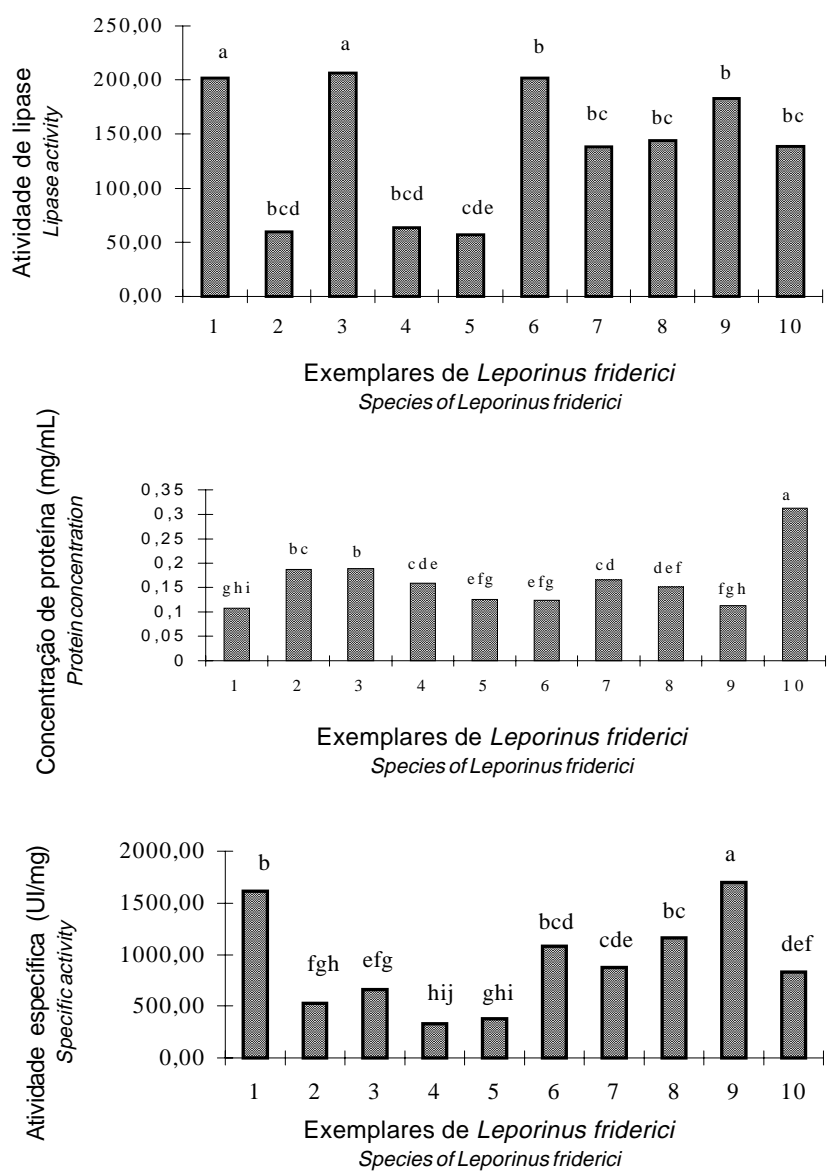

Figura 2 - Perfil das atividades, concentração de proteína e atividades específicas de lipase no quimo dos exemplares de piau, Leporinus friderici Médias seguidas pelas mesmas letras não diferem pelo teste $\mathrm{t}$, a $5 \%$ de probabilidade.

Figure 2 - Activity profile, protein concentration and lipase specific activity in the chyme of piau, Leporinus friderici species. Means followed by the same letters do not differ by $t$ test, at $5 \%$ probability.

Tabela 5 - Variação da atividade específica de lipase no quimo e sua relação com o peso e o comprimento nos exemplares do surubim, Pseudoplatystoma coruscans

Table 5 - Lipase specific activity variation in the chyme and its relation to weigth and length of surubim, Pseudoplatystoma coruscans species

\begin{tabular}{|c|c|c|c|c|c|}
\hline $\begin{array}{l}\text { Surubim } \\
\text { (exemplar) } \\
\text { (species) }\end{array}$ & $\begin{array}{l}\text { Peso } \\
(\mathrm{g}) \\
\text { Weight }\end{array}$ & $\begin{array}{c}\text { Comprimento } \\
(\mathrm{cm}) \\
\text { Length }\end{array}$ & $\begin{array}{c}\text { Atividade } \\
\text { específica } \\
\text { (U.I./mg) } \\
\text { Specificactivity }\end{array}$ & $\begin{array}{c}\text { Atividade/ } \\
\text { peso vivo (g) } \\
\text { (U.I./g) } \\
\text { Activity/liveweight }\end{array}$ & $\begin{array}{c}\text { Atividade/ } \\
\text { comprimento }(\mathrm{cm}) \\
\text { (U.I./cm) } \\
\text { Activity/livelength } \\
\end{array}$ \\
\hline 01 & $226,83 \mathrm{ij}$ & 30,00 cde & $195,996 \mathrm{efg}$ & 0,137 cde & $1,033 \mathrm{fgh}$ \\
\hline 02 & $304,09 \mathrm{hi}$ & $35,00 \mathrm{bc}$ & $722,380 \mathrm{~cd}$ & $0,419 \mathrm{~b}$ & 3,642 def \\
\hline 03 & $336,10 \mathrm{fgh}$ & $33,00 \mathrm{~cd}$ & $1287,708 \mathrm{a}$ & $0,926 \mathrm{a}$ & $9,428 \mathrm{a}$ \\
\hline 04 & 372,56 efg & $34,00 \mathrm{bc}$ & $248,419 \mathrm{efg}$ & 0,120 cde & 1,317 efg \\
\hline 05 & $410,27 \mathrm{def}$ & $32,50 \mathrm{~cd}$ & $635,701 \mathrm{cde}$ & $0,305 \mathrm{bcd}$ & $3,844 \mathrm{cde}$ \\
\hline 06 & $433,58 \mathrm{de}$ & $35,00 \mathrm{bc}$ & $1172,442 b$ & $0,314 b c$ & $3,895 \mathrm{bcd}$ \\
\hline 07 & $440,75 \mathrm{~cd}$ & $38,00 \mathrm{~b}$ & $261,125 \mathrm{efg}$ & $0,072 \mathrm{efg}$ & 0,830 ghi \\
\hline 08 & $612,03 \mathrm{bc}$ & $42,50 \mathrm{a}$ & $1043,753 \mathrm{bc}$ & $0,324 b c$ & $4,659 \mathrm{bc}$ \\
\hline 09 & $630,33 \mathrm{~b}$ & $42,00 \mathrm{a}$ & $1957,689 a$ & $0,430 \mathrm{~b}$ & $6,455 b$ \\
\hline 10 & $658,00 \mathrm{a}$ & $43,50 \mathrm{a}$ & $423,857 \mathrm{def}$ & $0,074 \mathrm{efg}$ & $1,113 \mathrm{fgh}$ \\
\hline $\mathrm{CV}(\%)$ & 31,97 & 12,61 & 73,10 & 77,43 & 73,75 \\
\hline
\end{tabular}

Em cada coluna, médias seguidas pelas mesmas letras não diferem pelo Teste t, a 5\% de probabilidade. In each column, means followed by the same letters do not differ by t test at $5 \%$ probability. 

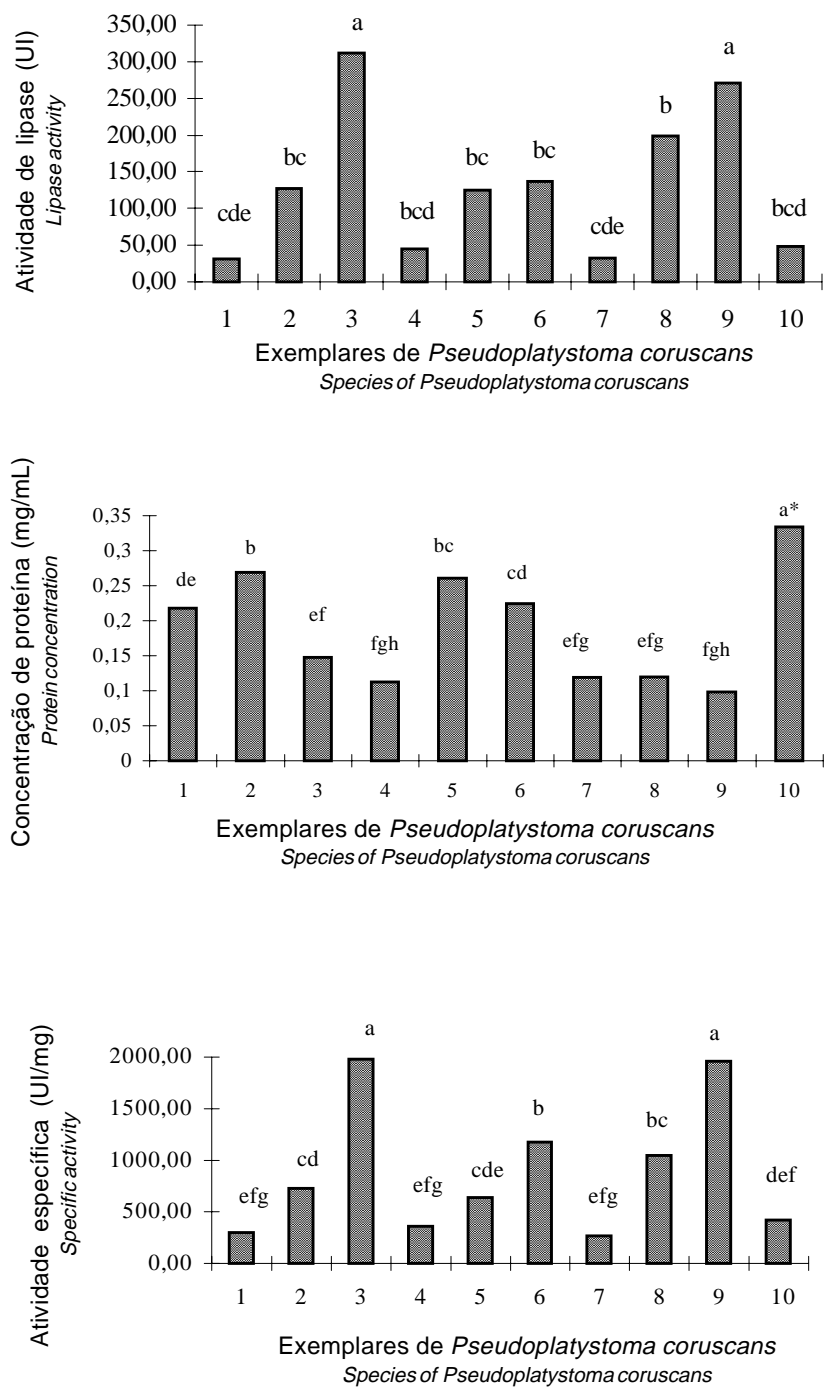

Figura 3 - Perfil das atividades, concentração de proteína e atividades de lipase no quimo dos exemplares de surubim, Pseudoplatystoma coruscans

* $\quad \begin{aligned} & \text { Médias seguidas pelas mesmas letras não } \\ & \text { diferem pelo teste t a } 5 \% \text { de probabilidade. }\end{aligned}$

* $\quad \begin{aligned} & \text { Médias seguidas pelas mesmas letras } \\ & \text { diferem pelo teste t a } 5 \% \text { de probabilidade. }\end{aligned}$

Figure 3 - Activity profile, protein concentration and lipase specific activity in the chyme of surubim, Pseudoplatystoma coruscans species.

Means followed by the same letters do not differ by t test, at $5 \%$ probability. a digestão de gordura que a piracanjuba, o que, nas condições em que se encontravam esses animais, pode estar relacionado com a deficiência nutricional, em razão do uso de ração comercial, que pode não ser adequada às exigências nutricionais dessa espécie. ABI-AYAD e KESTEMONT (1994) também verificaram maior atividade de lipase, em Carassius auratus, alimentados com ração artificial, quando comparada à dos que receberam dieta natural. Além disso, a alta atividade específica de lipase pode estar relacionada com a estrutura do intestino, que, conforme MENIN (1988), tem arranjo em N e, conseqüentemente, a espécie deve estar adaptada para alimentação com valores nutricionais intermediários entre os ictiófagos e os dos herbívoros ou iliófagos, apresentando comprimento intestinal intermediário entre as espécies iliófagas e carnívoras.

A maior atividade específica de lipase observada em piau, em relação à da piracanjuba, pode estar relacionada ao fato de o piau ter apresentado menor comprimento do intestino e arranjo intestinal menos complexo que o da piracanjuba, o que leva à redução da taxa de passagem do alimento, que passa a sofrer ação prolongada das enzimas digestivas. Por outro lado, um intestino mais longo, com arranjo mais complexo que o de piau, foi observado em piracanjuba por SEIXAS FILHO (1998); ou, ainda, como acontece em outros peixes Teleostei, segundo ALHUSSAINI (1949), a atividade máxima da lipase para o piau pode estar localizada no final da porção anterior do intestino, diminuindo gradualmente à sua porção final. Já a piracanjuba, também, pode apresentar atividade de lipase em outras regiões do tubo digestivo, conforme relatado por BORLONGAN (1990), que, estudando o milkfish, Chanos chanos, observou atividade dessa enzima no esôfago, indicando papel mais ativo desse órgão no processo digestivo.

Estudos futuros são necessários para que se 
SEIXAS FILHO et al.

Tabela 6 - Média das atividades específicas de lipase no quimo e da relação com o peso e o comprimento dos exemplares de piracanjuba, Brycon orbignyanus, piau, Leporinus friderici, e surubim, Pseudoplatystoma coruscans

Table 6 - Average of lipase specific activities in the chyme and its relation with weight and length of piracanjuba, Brycon orbignyanus, piau, Leporinus friderici, e surubim, Pseudoplatystoma coruscans species

\begin{tabular}{lcccc}
\hline $\begin{array}{l}\text { Espécie } \\
\text { Species }\end{array}$ & $\begin{array}{c}\text { Atividade } \\
\text { lipase } \\
\text { (U.I.) }\end{array}$ & $\begin{array}{c}\text { Atividade } \\
\text { específica } \\
\text { (U.I./mg) } \\
\text { Lipase activity }\end{array}$ & $\begin{array}{c}\text { Atividade } \\
\text { peso }(\mathrm{g}) \\
\text { (U.I./g) }\end{array}$ & $\begin{array}{c}\text { Atividade } \\
\text { comprimento }(\mathrm{cm}) \\
\text { (U.I./cm) }\end{array}$ \\
\hline Piau & $2.209,799 \mathrm{a}$ & $1347,824 \mathrm{a}$ & $1,553 \mathrm{a}$ & $11,582 \mathrm{a}$ \\
Surubim & $131,917 \mathrm{~b}$ & $793,765 \mathrm{~b}$ & $0,315 \mathrm{c}$ & $3,621 \mathrm{~b}$ \\
Piracanjuba & $0,2815 \mathrm{c}$ & $1,886 \mathrm{c}$ & $0,502 \mathrm{~b}$ & $0,430 \mathrm{c}$ \\
CV $(\%)$ & 105,04 & 102,06 & 124,33 & 123,73 \\
\hline
\end{tabular}

Em cada coluna, médias seguidas pelas mesmas letras não diferem pelo teste t, a $5 \%$ de probabilidade.

In each column, means followed by the same letters do not differ by $t$ test at $5 \%$ probability.

possa obter maiores entendimentos sobre a fisiologia digestiva dessas espécies, a fim de que possa dar subsídios a uma recomendação mais segura de uma dieta que melhor atendam às necessidades do animal e aos interesses zootécnicos.

\section{Conclusões}

A grande diferença da atividade específica de lipase apresentada entre o piau e o piracanjuba sugere estreita ligação com a morfometria e o complexo arranjo das pregas da mucosa dos intestinos médio e posterior, ou inadequado balanceamento da ração comercial utilizada no arraçoamento dessas espécies.

A atividade específica de lipase apresentada pelo piau foi mais próxima à de um peixe carnívoro que um onívoro.

Os valores de atividade específica maiores para o piau em relação ao surubim sugere balanceamento inadequado da ração para a espécie onívora.

\section{Referências Bibliográficas}

ABI-AYAD, A., KESTEMONT, P. 1994. Comparison of the nutritional status of goldfish (Carassius auratus) larvae fed with live, mixed or dry diet. Aquaculture, 128:163-176.

AGRAWALL, V.P., SASTRY, K.V., KAUSHAB, S.K.S. 1975. Digestive enzymes of three teleost fishes. Acta Physiol. Hung, 46:93-98.

AL-HUSSAINI, A.H. 1947.The feeding habit and the morphology of the alimentary tract of some teleosts living in the neighbourhood of the Marine Biological Station, Ghardaqa, Red Sea. Publ. Mar. Biol. Stat., 5:4-61.

ANDREWS, W. 1954. Textbook of comparative histology, Oxoford University Press. p.302.

BORLONGAN, I.G. 1990. Studies on the digestive lipases of milkfish, Chanos chanos. Aquaculture, 89:315-325.

CASTAGNOLLI, N. 1997. Espécies nativas próprias para a piscicultura. In: SIMPÓSIO SOBRE MANEJO E NUTRI-
ÇÃO DE PEIXES. 1997, São Paulo. Anais... Piracicaba: Colégio Brasileiro de Nutrição Animal: 117-130.

CASTAGNOLLI, N. 1992. Piscicultura intensiva e sustentável de espécies nativas brasileiras. In: Piscicultura de água doce. Jaboticabal: FUNEP. 189p.

CHERRY, I.S., CRANDALL, L.A. 1932. Am. J. Physiol., 100:266.

CHESLEY, L. 1934. The concentration of proteases, amylase and lipase in certain marine fishes. Bio. Bull., 66:133-144.

CYRINO, J.E.P. 1984. Digestibilidade da proteína de origem animal e vegetal pelo matrinxã, Brycon cephalus, GÜNTHER, 1869 (Teleostei, Characoidei, Caracidae). Manaus, AM: INPA/FUA, 1984. 39p. Dissertação (Mestrado em Biologia de Água Doce e Pesca Interior) - INPA/FUA,

INFANTE, J.L.Z, CAHU, C.L. 1994. Influence of diet on pepsin and some pancreatic enzymes in sea bass (Dicentrarchus labrax) larvae. Great Britain. Comp. Biochem. Physiol., 109A(2):209-212.

KAWAI, S., IKEDA, S. 1972. Studies on digestive enzymes of fisheries. II - Effect of dietary change the activities of the digestive enzymes in carp intestines. Bull. Jap. Soc. Sci. Fish., 38:265-27.

KITAMIKADO, M., TACHINO, S. 1961. Studies on the digestive enzymes of the rianbow trout - III. Esterases. Chem. Abstr., 55:5789C.

KUZ'MINA, V.V. 1996. Influence of age on digestive enzyme activity in some freshwater teleosts. Aquaculture, 148:25-37.

MENIN, E. Anátomo-histologia funcional comparativa do aparelho digestivo de seis Teleostei (Pisces) de água doce. São Paulo: USP, 1988. 557p. Tese (Doutorado) - Universidade de São Paulo, 1988.

MENIN, E., MIMURA, O.M. 1992. Anatomia funcional da cavidade bucofaringeana de Prochilodus marggravii (Wallbaum, 1792) e Prochilodus affinis Reinhardt, 1874 (Characiformes, Prochilodontidae). Rev. Ceres, 39 (226):506-527.

NAGASE, G. 1964. Contribution to the phisiology of digestion in Tilapia mossambica (PETERS): digestive enzymes and effect on their activity. Z. Vgl. Phisiol., 49:270-284.

NAKAGAWA, H., TAKEDA, T., UMINO, T. et al. 1995. Effects os feeding regime on biometric parameters and hepatic enzymes activities of young red sea bream, Pagrus major. Hiroshima, J. Fac. Appl. Biol. Sci., 34:167-178.

NIKOLSKY, G.V. 1963. The ecology of fishes. London, Academic Press. 325p.

PEZZATO, L.E.O 1997. estabelecimento das exigências nutricionais das espécies de peixes cultivadas. In: SIMPÓSIO 
14 Rev. bras. zootec.

SOBRE MANEJO E NUTRIÇÃO DE PEIXES. 1997, São Paulo. Anais...Piracicaba: Colégio Brasileiro de Nutrição Animal, p.45-62, 1997.

PREJS, A. 1981. Metodos para el estudio de los alimentos y las relaciones troficas de los peces. Caracas: Universidad Central de Venezuela y Universidad de Varsovia. 129p.

REIMER, G. 1982. Studies on the enzymatic activities in the gastrointestinal of the Brycon metanoptoris. J. Fish Biol., 21:637-642.

RICKER, W.E. 1968. Methods for assessment of fish production in fresh waters. Oxford: Blackwell Scientific Publications. 313p.

SABAPATHY, U., TEO, L.H. 1993. A quantitative study of some digestive enzymes in the rabbitfish, Siganus canaliculatus and the sea bass, Lates calcarifer. J. Fish Biol., 42:595-602.

SASTRY, K.V. 1974a. Distribution of lipase in the digestive system of two teleost fishes. Acta Histochem., 48S:320-325.

SASTRY, K.V. 1974b. Histochemical localization of esterase and lipase in the digestive system of two teleost fishes. Acta Histochem., 51S:18-23.
SEIXAS FILHO, J.T. 1998. Anatomia funcional e sistemas enzimático e endócrino do intestino de Teleostei (Pisces) de água doce com hábitos alimentares diferentes. Viçosa, MG: UFV, 1998. 175p. Tese (Doutorado em Zootecnia) - Universidade Federal de Viçosa. 1998.

SABAPATHY, U., TEO, L.H. 1993. A quantitative study of some digestive enzymes in the rabbitfish, Siganus canaliculatus and the sea bass, Lates calcarifer. British Isles, J. Fish Biol., 42:595-602.

SMITH, L.S. 1980. Digestion in teleost fish. In: Lectures presented at the FAO/UNPD training course in fish feed technology, ADCP/REP/80/11. p.3-17.

WARBURG, O., CHRISTIAN, W. 1941. Isohering und kristallisation des gõrungs ferments enolase. Biochem. Z., 310:384-421.

Recebido em: 17/03/99

Aceito em: 23/06/99 